\title{
Transient Acute Myeloid Leukemia in a Newborn with Down's Syndrome
}

\author{
ZSM HAQUE $^{\mathrm{a}}$, N JAHAN ${ }^{\mathrm{b}}$, BK RAHA ${ }^{\mathrm{c}}, \mathrm{M} \mathrm{HASAN}^{\mathrm{d}}$, M BEGUM $^{\mathrm{e}}$, AWS ROB $^{\mathrm{f}}$, \\ S YASMIN $^{\mathrm{g}}$, SKAHASNAT ${ }^{\mathrm{h}}$, TFARHANA ${ }^{\mathrm{i}}$
}

\begin{abstract}
Summary:
We report the case of a newborn with Down's syndrome associated with transient acute myeloid leukemia (AML). The leukemic presentation resolved spontaneously without treatment just 4 weeks after birth. A 2 days old newborn presented with respiratory distress, lethargy, poor suck and mild hepatosplenomegaly with features of Down's syndrome. Total white cell count was 144,000/cmm with blasts 92\%. Other Septic work up was negative. Peripheral blood smear revealed hyper leukocytosis and presence of blast cells. Flow cytometric analysis revealed acute myeloid leukemia. The peripheral blast cells
\end{abstract}

Introduction:

Down's syndrome (DS) or constitutional trisomy 21 was linked to leukemia for the first time in a case report published in $1930^{1}$. The incidence of acute leukemia in patients with DS is 14 times higher than in unaffected children, and the disease is mainly myelogenous ${ }^{2}$. Transient abnormal myelopoesis is almost unique to Down's syndrome, and probably occurs in about $10 \%$

a. Prof. Zabrul SM Haque, Professor of Neonatology, Ad-din Medical College Hospital, Dhaka, Bangladesh.

b. Dr. Nasim Jahan, Assistant Professor of Neonatology, Addin Medical College Hospital, Dhaka, Bangladesh.

c. Dr. Biplob Kumar Raha, Assistant Professor of Neonatology, Ad-din Medical College Hospital, Dhaka, Bangladesh.

d. Dr. Mahmuda Hassan, Associate Professor of Neonatology, Ad-din Medical College Hospital, Dhaka, Bangladesh.

e. Dr. Mariam Begum, Assistant Professor of Neonatology, Ad-din Medical College Hospital, Dhaka, Bangladesh.

f. Dr. AWS Rob, Assistant Professor of Neonatology, Ad-din Medical College Hospital, Dhaka, Bangladesh.

g. Dr. Sabina Yasmin, Indoor Medical Officer of Neonatology, Ad-din Medical College Hospital, Dhaka, Bangladesh.

h. Dr. SKA hasnat, Indoor Medical Officer of Neonatology, Ad-din Medical College Hospital, Dhaka, Bangladesh.

i. Dr. Tasnim Farhana, Indoor Medical Officer of Neonatology, Ad-din Medical College Hospital, Dhaka, Bangladesh.

Address of Correspondence: Dr. Nasim Jahan, Assistant professor, Department of Neonatology, Ad-din Medical College Hospital, Dhaka. Mob.01757078207, Email: njahan.jesy@gmail.com

Received: 25 September 2013

Accepted: 6 April 2016 persisted for 4 weeks, after which the hemogram and the differential WBC count returned to normal and a final diagnosis of transient acute myeloid leukemia was made. Only few cases of congenital leukemia with Down's syndrome have been reported in the literature. In Down's syndrome, AML whether transient or not, generally shows cytogenetic and molecular aspects that differ from those of adult acute leukemias.

Key words: Down's syndrome, Transient myeloproliferative disease, Transient leukemia.

(J Bangladesh Coll Phys Surg 2016; 34: 213-217)

of newborn infants. Because the leukemic picture resolves spontaneously, the disorder has been called transient myeloproliferative disorder (TMD), transient blastemia, or transient abnormal myelopoesis. Now the disease is commonly referred to as "transient leukemia" (TL). Typically, TL is diagnosed in clinically normal children with DS via the casual detection of leukocytosis with circulating blast cells. The characteristic feature of transient leukemia in DS patients is that the TL disappears on its own without treatment in just a few months. The typical and persistent spontaneous remission of the leukemic presentation in 4 to 6 weeks suggests a transient disruption of the granulocytopoietic system $^{3}$. The TMD is a clonal disease characterized by accumulation of immature megakaryoblast in fetal liver and peripheral blood, a picture indistinguishable from $\mathrm{AL}^{4,5,6}$. We report the case of Down's syndrome with transient acute leukemia presented in NICU at Ad-din Medical College Hospital.

\section{Case Report:}

A term female baby born to 25 years old mother of non consanguineous marriage by Lower Segment Ceasarian Section (LUCS) due to maternal Gestational Diabetes Mellitus (GDM). There was no history of cytotoxic drug intake or exposure to radiation during antenatal period. Special emphasis was given to elicit the history of maternal fever, rash or lymphadenopathy in the 
mother in the first trimester to rule out intrauterine infection. Her birth weight was $3.3 \mathrm{~kg}$, and APGAR score was $8 / 10$ and $9 / 10$ at one and five minutes respectively. On $2^{\text {nd }}$ day the baby developed difficulty in breathing. Her respiratory rate was $70 / \mathrm{min}$, Heart rate was $180 / \mathrm{min}$, no cyanosis was observed and $\mathrm{SpO}_{2}$ $>95 \%$ with $2 \mathrm{~L} \mathrm{O}_{2}$ by nasal cannula, and temperature was with in normal limit. No lymph nodes were palpable. Physical examination revealed features of Down's syndrome (dysmorphic facies, epicanthic folds, hypotonia, and simian crease in hands). She was tachypnic, berath sound vesicular with no added sound. Her blood pressure of all four limbs were within normal limits, there was a systolic murmur over left lower sternal area. Abdomen was distended with hepato-splenomegaly, Liver was palpable $4 \mathrm{~cm}$ from right costal margin at mid clavicular line and spleen was $3 \mathrm{~cm}$ below left costal margin. A clinical diagnosis of Down's syndrome with congenital heart disease was made. Total white cell count was $145000 / \mathrm{cmm}$ with blasts 92\%. Peripheral smear revealed Anisocytic with anisochromic RBC morphology. WBC was increased in number with shift to the lef, majority cells were immature mononuclear cells, morphologically resembling blast, Platelet was normal initially then decreased. Flow cytometric analysis reveals acute myloid leukemia; overall findings are suggestive of acute myeloid leukemia without maturation. Exchange transfusion was done on day 8. Total WBC count was reduced to $37,620 / \mathrm{cmm}$. The infant was followed and monitored without any specific treatment for leukemia. The peripheral blast cells persisted for 4 weeks, after which the hemogram and the differential WBC count returned to normal (WBC- 19,770/ cu mm, $\mathrm{Hb} 8.2$, PC- $68,000 / \mathrm{cu} \mathrm{mm}$ ), and peripheral blast cells disappeared. Respiratory distress improved in course of time with restriction of the fluid and lowering of leucocytes number possibly had affect on improvement of vitals. The case was labeled as Transient Acute Myeloid leukaemia (AML). Karyotyping showed Triosomy 21. Bone marrow morphology and Cytogenetic studies and serial blood counts were suggested. The baby also had congenital heart disease and intestinal obstruction. Surgical consultation was taken for double bubble appearance of the abdominal X-ray and surgical intervention could not be taken due to poor general condition of the baby. We also planned to do the Echo cardiogram wich finally could not be performed in our center for the mechanical dysfunction of the machine.

Table-I

\begin{tabular}{|c|c|c|c|c|c|c|c|c|c|}
\hline \multicolumn{10}{|c|}{ Serial lab report } \\
\hline Investigations & Day 1 & Day 4 & Day 7 & $\begin{array}{c}\text { Day } 9 \\
\text { ('post } \\
\text { exchange) }\end{array}$ & Day 12 & Day 15 & Day 19 & Day 22 & Day 32 \\
\hline $\mathrm{WBC}(/ \mathrm{cmm})$ & 121,91 & 134,600 & 145,000 & 37,620 & 60,500 & 40,970 & 24,530 & 19,770 & 13,140 \\
\hline Atypical cell & $70 \%$ & $82 \%$ & $92 \%$ & $50 \%$ & $50 \%$ & $30 \%$ & $25 \%$ & $0 \%$ & \\
\hline $\mathrm{Hb} \%$ & 14.4 & 14.3 & 12.5 & 11.9 & 14.5 & 13.8 & 11.6 & 8.2 & 10.1 \\
\hline Platelets (/cmm) & 283,000 & 220,000 & 196,000 & 141,000 & 144,000 & 187,000 & 35,000 & 68,000 & 26,000 \\
\hline X-ray abdomen & \multicolumn{9}{|l|}{$\begin{array}{l}\text { Double } \\
\text { bubble } \\
\text { shadow }\end{array}$} \\
\hline USG of abdomen & \multicolumn{9}{|c|}{ Hepatic calcification, Bilateral echogenic kidney } \\
\hline Flow cytometry & \multicolumn{9}{|c|}{ Blasts are positive for CD34, CD33, CD41, CD61, glycophorin A, and often CD7 and CD36. } \\
\hline
\end{tabular}




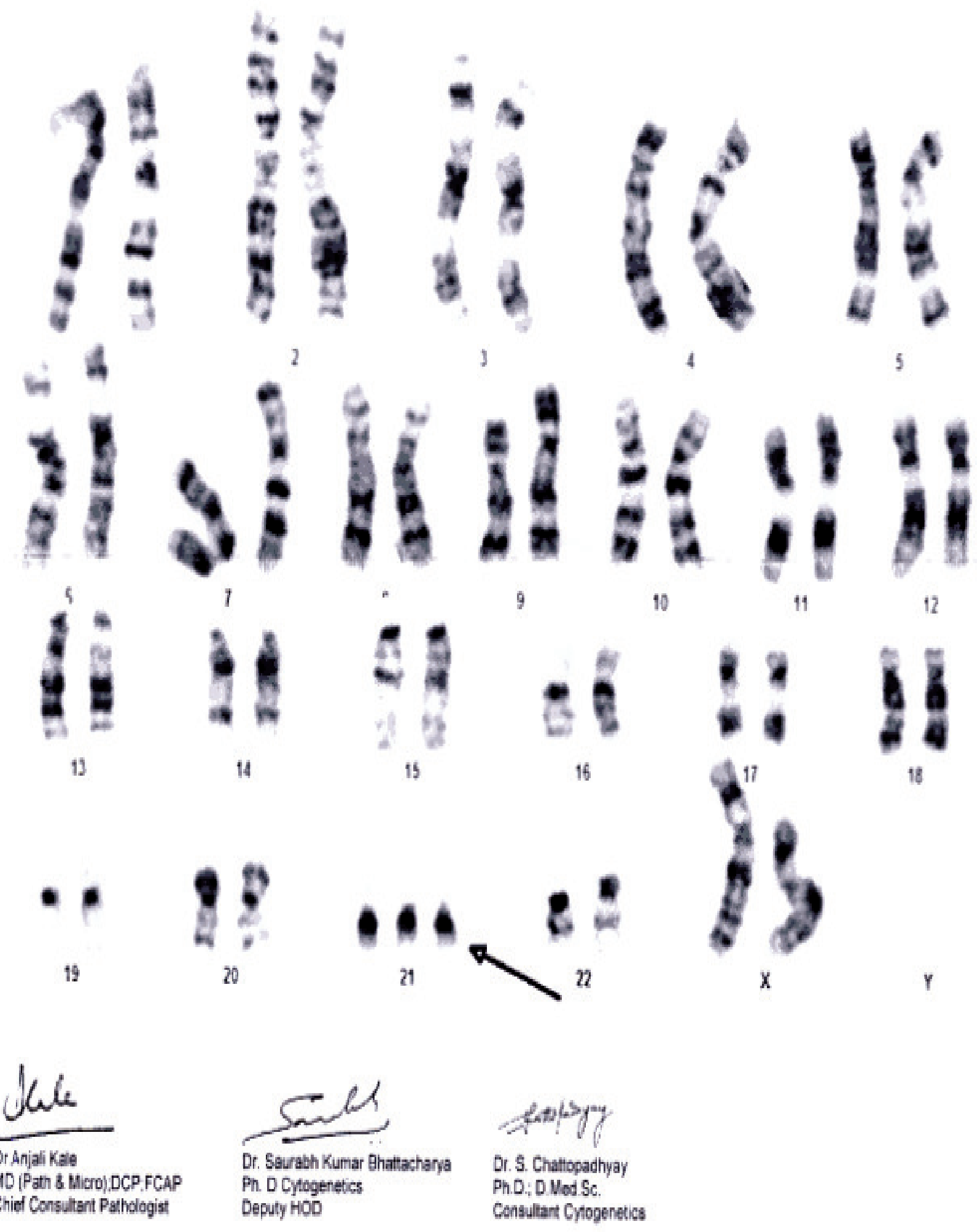

Fig. -1: Chromosome analysis (Karyotype): Trisomy 21 

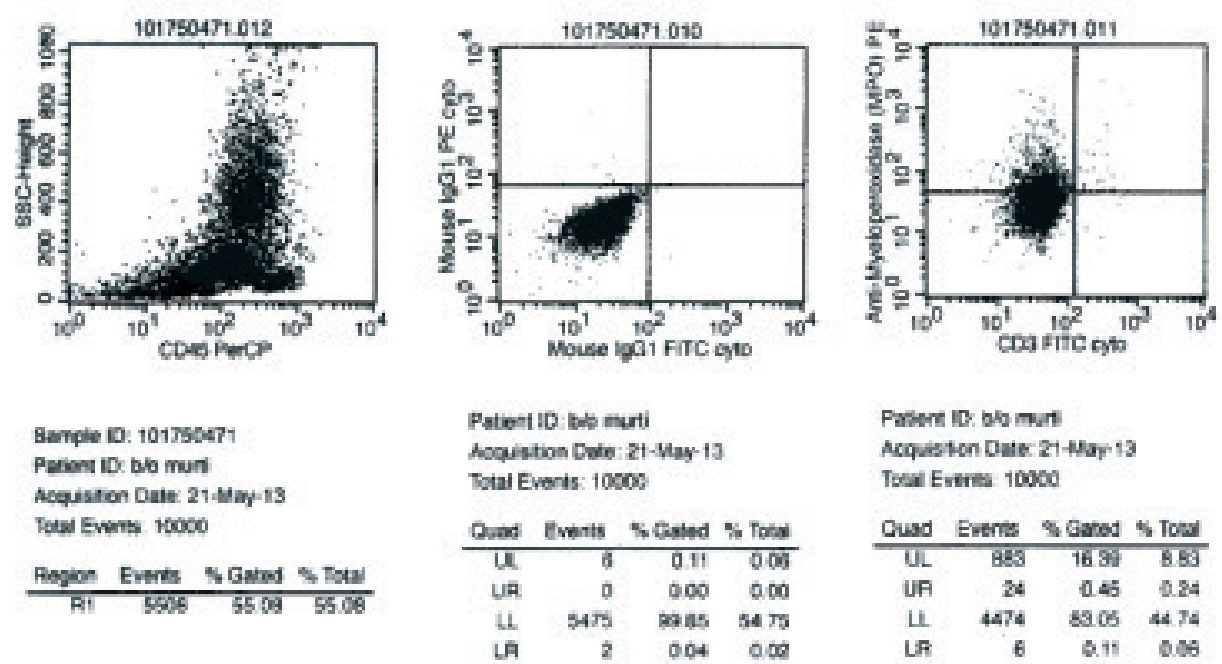

\section{Pabere iD. blo munt \\ Acquiston Date: $21+$ May 13} Toeal Everes 10000

\begin{tabular}{|c|c|c|c|}
\hline Quad & Events & \$ Ganed & $\$$ Tot \\
\hline UL & BEB & 168 & 8.53 \\
\hline UA & 24 & 0.45 & 0.24 \\
\hline $\mathbf{u}$ & 4474 & 89.05 & 4474 \\
\hline LR & 6 & Q.11 & 0,06 \\
\hline
\end{tabular}
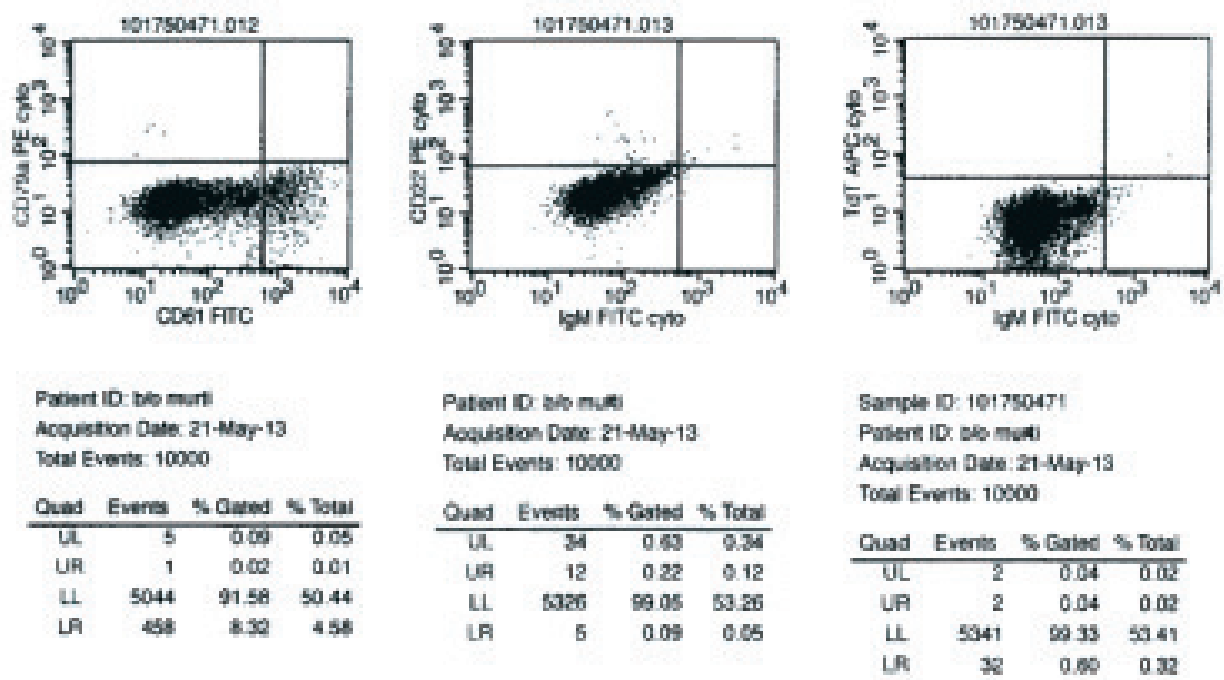

Fig.-2: Flow cytometry:

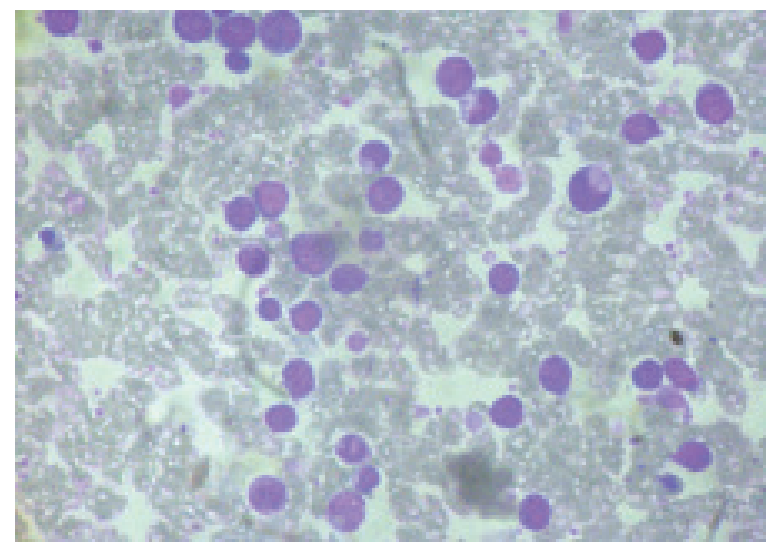

Fig.-3: Peripheral Blood film showing Blast cells 


\section{Discussion:}

The diagnosis of TMD usually occurs during the first weeks after birth and is observed as hydrops fetalis. The elevated blood count associated with hepatomegaly is the common symptom in an asymptomatic neonate. Infants with TMD can also display occasionally jaundice and bleeding diastheses, respiratory distress coupled with ascites, pleural effusion, signs of heart failure, and skin infiltrates. There is megakaryocytic infiltration and liver fibrosis, likely caused by excess cytokines secreted from the megakaryoblasts. The full clinical TMD may develop only at the second or third week of life. Laboratory tests are significant for either thrombocytosis or thrombocytopenia accompanied by elevated leukocytes with excess of blasts. This findings consistant with our case. The cause of thombocytopenia after two weeks of life could be due to direct effect of leukemia on the bone morrow of the baby or could be sepsis which was clinicaly evident with negative blood culture result. Also hypersplenia could have contribution to thrombocytopenia in our case. The differential diagnosis includes leukoerythroblastic reaction associated with prematurity, sepsis, or asphyxia. However, the blasts of TMD usually persist for several weeks, and GATA1 mutations are invariably found ${ }^{5}$. Flow cytometry reveals that blasts are positive for CD34, CD33, CD41, CD61, glycophorin A, and often CD7 and CD36 ${ }^{5,7}$. Savasan \& Ravindranath (2003) observed that blasts of DS children with AMKL express CD36, in contrast to the low or no expression of CD36 in AML without $\mathrm{DS}^{8}$. If $25 \%$ of blast cells are not detected, the diagnosis of AMKL can be given by the megakaryocytic markers CD41, CD61 and CD42a. The immunophenotype of the blasts in AMKL is generally similar to TMD, except that the percentage of CD34 cells may be lower in AMKL 5,6 . In our case flow cytometric analysis reveals CD13, CD33, CD36, CD117, MPO myeloid markers \&CD34 \& CD45 are positive. Several treatment approaches have been used, including exchange transfusion, leukapheresis, and low dose cytarabin ${ }^{9}$. Exchange transfusion was done in our case. Although baby recovered from leukemia but was expired from sepsis in another hospital at 56 days of age.

\section{Conclusion:}

The course of congenital leukemia is one of rapid deterioration and death from hemorrhage and infection. While diagnosing congenital leukemia, the condition of transient leukemia in Down's syndrome should always be kept in mind as the later entity is associated with complete remission.

\section{References:}

1. Cannon HE. Acute lymphatic leukemia: report of a case in an eleventh month Mongolian idiot. New Orleans Med Surg J 1930; 94(3):289-93.

2. Kojima S, Matsayama T, Sato T. Down's syndrome and acute leukemia in children: an analysis of phenotype by use of monoclonal antibodies and electron microscopic platelet peroxidase reaction. Blood 1990;76:2348-2353.

3. Coulombel L, Derycke M, Villeval JL. Characterization of the blast cell population in two neonates with Down's syndrome and transient myeloproliferative disorder. $\mathrm{Br} \mathrm{J}$ Haematol 1987;66: 69-76.

4. Hitzler J.K, Cheung J, Li Y, Scherer S.W, Zipursky A. GATA1 mutations in transient leukemia and acute megakaryoblastic leukemia of Down's syndrome Blood 2003;101(11):4301-4304.

5. Malinge S, Izraeli S, Crispino J.D. Insights into the manifestations, outcomes, and mechanisms of leukemogenesis in Down's syndrome. Blood 2009; 113(12):2619-2628.

6. Pine S.R, Guo Q, Yin C, Jayabose S, Druschel C.M, Sandoval C. Incidence and clinical implications of GATA1 mutations in newborns with Down's syndrome. Blood 2007; 110(6): 2128-2131.

7. Langebrake C, Creutzig U, Reinhardt D. Immunophenotype of Down's syndrome acute myeloid leukemia and transient myeloproliferative disease differs significantly from other diseases with morphologically identical or similar blasts. Klinische Pädiatrie 2005 ;217(3): 126-134.

8. Savasan S.B.S, Ravindranath Y. Cd36 Expression Is Associated With Superior In Vitro Ara-C Sensitivity In Acute Megakaryocytic Leukemia With And Without Down's Syndrome.Medical and Pediatric Oncology 2003;41(10):274-275.

9. Al-Kasim F, Doyle JJ, Massey GV. Incidence and treatment of potentially lethal diseases in transient leukemia of Down's syndrome: Pediatric Oncology Group Study. J Pediatr Hematol Oncol 2002;24(1) : 9-13. 